\title{
SCientific Oral Presentation Abstracts
}

diomic features (first-order histogram $(\mathrm{FOH})$, graylevel co-occurrence matrix (GLCM), run-length matrix (RLM), and Gabor filters) were calculated from mp-MRI. Statistical analysis was performed using receiver-operating-characteristic curve analysis for feature filtering, linear discriminant analysis (LDA) for feature extraction, and leave-one-out cross-validation for evaluation of the method in the differentiation of benign and malignant lesions.

Results: An accuracy of $96.6 \%$ was achieved for discriminating benign and malignant prostate lesions from a subset of texture features derived from ADC and DCE maps (radiomics-based method) with sensitivity and specificity of $100 \%$ and $85.7 \%$, respectively.

Conclusion: A radiomic quantification method based on T2-weighted images, ADC maps, and quantitative and semiquantitative DCE maps can discriminate benign from malignant prostate lesions with promising accuracy. This method is helpful to avoid unnecessary biopsies in patients and may provide information for CAD systems for the classifications of prostate lesions as an auto-detection technique.

\section{-10.5812/IRANJRADIOL.99136}

\section{An Efficient Framework for Accurate Arterial Input Selection in DSC-MRI of Glioma Brain Tumors}

Hossein Rahimzadeh ${ }^{1}$; Salman Rezaie Molood ${ }^{1}$; Anahita Fathi Kazerooni ${ }^{1}$; Hamidreza Saligheh $\operatorname{Rad}^{1,}$ *

${ }^{1}$ Quantitative MR Imaging and Spectroscopy Group (QMISG), Research Center for Molecular and Cellular Imaging, Tehran University of Medical Sciences, Tehran, Iran

${ }^{*}$ Corresponding author: Quantitative MR Imaging and Spectroscopy Group (QMISG), Research Center for Molecular and Cellular Imaging, Tehran University of Medical Sciences, Tehran, Iran. Email: hamid.saligheh@gmail.com

\section{Abstract}

Background:Arterial input function (AIF) accurate extraction is an important step in the quantification of cerebral perfusion hemodynamic parameters using dynamic susceptibility contrast magnetic resonance imaging (DSC-MRI).

Objectives: In this study, using machine learning methods, an optimal automatic algorithm was developed to accurately detect AIF in DSC-MRI of glioma brain tumors with a new pre-processing method.

Methods: DSC-MR images of 43 patients with glioma brain tumors were retrieved retrospectively. Our proposed method consisted of a pre-processing step to remove non-arterial curves such as tumorous, tissue, noisy, and partial-volume affected curves and a clustering step through agglomerative hierarchical $\mathrm{AH})$ clustering method to cluster the remaining curves. The performance of automatic AIF clustering was compared with the performance of manual AIF selection by an experienced radiologist, based on curve shape parameters, i.e., maximum peak (MP), full-width-at-half-maximum (FWHM), M (= MP / (TTP $\times$ FWHM)), and root mean square error (RMSE).

Results: The mean values of AIFs shape parameters were compared with those derived from manually selected AIFs by a two-tailed Paired t-test. The results showed statistically insignificant differences in MP, FWHM, and M parameters and lower RMSE, confirming the resemblance of the selected AIF with the gold standard. The intraclass correlation coefficient and percentage coefficient of variation showed a better agreement between manual AIF and our proposed AIF selection method rather than previously proposed methods.

Conclusion: The results of the current work suggest that by using efficient preprocessing steps, the accuracy of automatic AIF selection could be improved and this method appears to be promising for efficient and accurate clinical applications.

\section{• 10.5812/IRANJRADIOL.99139}

\section{Automatic Myocardial Segmentation in Four-Chamber View Echocardiography Images}

Shakiba Moradi'; Mostafa Ghelich Oghli², *; Azin Alizadehasl; Ali Shabanzadeh ${ }^{2}$

1'Sharif University of Technology, Tehran, Iran
${ }^{2}$ Intelligent Imaging Technology Research Center, Med Fanavarn Plus
Co., Karaj, Iran
${ }^{3}$ Rajaie Cardiovascular Medical and Research Center, Iran University
of Medical Science, Tehran, Iran
${ }^{*}$ Corresponding author: Intelligent Imaging Technology Research Cen-
ter, Med Fanavarn Plus Co., Karaj, Iran.Email:m.g31_mesu@yahoo.com

\section{Abstract}

Background:Most quantitative features in analyzing echocardiography images are elicited from the shape of different parts of the heart. One of the challenging tasks in this area is detecting the border between the left ventricle and its wall. Segmentation that is a process to extract the shape of objects in an image is a way to have a better observation of epicardial and endocardial parts of the left ventricle. Today, manual segmentation is performed by 